\title{
Hominin cranial fragments from Milner Hall, Sterkfontein, South Africa
}

AUTHORS:

Amélie Beaudet ${ }^{1,2}$

Jason L. Heaton ${ }^{3,4.5}$

Ericka N. L'Abbé2

Travis R. Pickering $4,5,6$

Dominic Stratford ${ }^{1}$

\section{AFFILIATIONS:}

'School of Geography,

Archaeology and Environmenta

Studies, University of the

Witwatersrand, Johannesburg,

South Africa

2Department of Anatomy,

University of Pretoria, Pretoria,

South Africa

${ }^{3}$ Department of Biology, Birmingham-Southern College,

Birmingham, Alabama, USA

${ }^{4}$ Evolutionary Studies Institute, University of the Witwatersrand Johannesburg, South Africa

${ }^{5}$ Plio-Pleistocene Palaeontology

Section, Department of

Vertebrates, Ditsong National

Museum of Natural History,

Pretoria, South Africa

'Department of Anthropology,

University of Wisconsin,

Madison, Wisconsin, USA

CORRESPONDENCE TO:

Amélie Beaudet

EMAIL:

beaudet.amelie@gmail.com

\section{DATES:}

Received: 01 July 2018

Revised: 15 Aug. 2018

Accepted: 15 Aug. 2018

Published: 27 Nov. 2018

\section{KEYWORDS:}

Cradle of Humankind; late Pliocene-early Pleistocene; cranial thickness; diploë; Homo

\section{HOW TO CITE:}

Beaudet A, Heaton JL, L'Abbé EN, Pickering TR, Stratford D. Hominin cranial fragments from Milner Hall, Sterkfontein, South Africa. S Afr J Sci. 2018;114(11/12), Art. \#5262, 6 pages. https://doi. org/10.17159/sajs.2018/5262

\section{ARTICLE INCLUDES: \\ $\times$ Supplementary material \\ $\times$ Data set}

(c) 2018. The Author(s). Published under a Creative Commons Attribution Licence.

The Sterkfontein Caves site is one of the richest early hominin localities in Africa. In addition to significant fossil assemblages from Members 2 and 4 of the Sterkfontein Formation, recent excavations have revealed hominin-bearing sedimentary deposits in the lesser-known Milner Hall. We describe two hominin cranial fragments excavated from the Milner Hall in 2015 and present the results of a highresolution microtomographic-based approach to diagnosing the anatomical and taxonomical origin of these specimens. Based on external morphology, StW 671 and StW 672 are identified as frontal and occipital fragments, respectively. Our non-invasive bi-dimensional quantitative investigation of the two cranial fragments reveals a mean cranial thickness of $8.8 \mathrm{~mm}$ for StW 671 and of $5.6 \mathrm{~mm}$ for StW 672 , and a contribution of the diploic layer to the cumulative cranial thickness that is less than $50 \%$. While the mean cranial thickness of StW 671 falls within the range for Homo, the relative proportion of the diploë in both StW 671 and StW 672 is lower than that found in Australopithecus (>60\%) and extant humans (>50\%). Accordingly, in terms of both cranial thickness and inner structural organisation, the Milner Hall hominins combine derived and unique traits, consistent with the condition of other postcranial and dental material already described from the deposit. Moreover, our study opens interesting perspectives in terms of analysis of isolated cranial fragments, which are abundant in the hominin fossil record.

Significance:

- The Sterkfontein Caves have widely contributed to our understanding of human evolution.

- Besides the well-known Members 4 and 2, where the iconic 'Mrs Ples' and 'Little Foot' have been found, in this study we suggest that the Milner Hall locality represents an additional, stratigraphically associated source of not only fossil hominins, but also Oldowan stone tools.

- In particular, we describe for the first time two cranial fragments, StW 671 and StW 672, identified as frontal and occipital bones, respectively.

- Our microtomographic-based analysis of these materials reveals some affinities with Homo combined with unique characters.

- In this context, our study suggests an intriguing mosaicism consistent with the description of the two fossil hominins found in the Milner Hall.

\section{Introduction}

Milner Hall $(\mathrm{MH})$ is a deep underground chamber of the Sterkfontein Caves (South Africa) that extends about $100 \mathrm{~m}$ in a roughly east-west direction. Two hominin fossils, a molar and a proximal phalanx, excavated from the T1 depositional unit of the Central Underground Deposits excavation site in the Milner Hall (STK-MH1) have already been described. ${ }^{1}$ The complex stratigraphic context of the Milner Hall fossiliferous depositional sequence, in which an early distal accumulation of the 3.67-Ma-old Member 2 (T3) and 2.18-Ma-old Oldowan artefact-bearing sediments from Member 5 (T2) contribute to the formation of $\mathrm{T}^{2,3}$, affords that potentially Australopithecus, Paranthropus and early Homo might be represented in the unit. Interestingly, the description and metric analyses of the two first hominin specimens excavated from $\mathrm{T} 1$ suggest an enigmatic mix of unique, primitive and derived morphological traits, with potential morphological affinities with the genus Homo.

With hominin remains dated potentially to either $3.67 \mathrm{Ma}$ or $2.18 \mathrm{Ma}$, the Milner Hall fossil assemblage may contribute to ongoing debates about hominin morphological and taxonomic diversity at Sterkfontein during the late Pliocene and early Pleistocene. In particular, because of the poorly known degree of intraspecific variation in the Australopithecus hypodigm, the Sterkfontein hominin fossil assemblages have been the focus of longstanding discussions regarding the presence of one or two Australopithecus species. ${ }^{4,5}$ In addition, the specieslevel diagnosis of purported early Homo remains from Member 5 of the Sterkfontein Formation is contentious. ${ }^{6-8}$ In particular, the re-attribution of hominin remains previously assigned to early Homo or Australopithecus (e.g. StW 53$)^{8}$, and the fragmentary nature of fossil specimens identified as early Homo (e.g. SK 847) ${ }^{9}$, complicate our understanding of early human diversity in South Africa. In this context, the Milner Hall deposits have the potential to provide further evidence to critically assess hominin palaeobiodiversity and the taxonomic context of the Sterkfontein hominin-bearing deposits.

FUNDING:

AESOP+ Programme; Claude Leon Foundation; DST-NRF Centre of Excellence in Palaeosciences; French Institute of South Africa; NRF African Origins Platform; Palaeontological Scientific Trust (PAST) 
Here, we report on two additional hominin fossils excavated from STK$\mathrm{MH} 1$ and discuss efforts to assign them taxonomically.

\section{Material and methods}

As comparative material, we investigated four South African hominin cranial specimens from Sterkfontein (Sts 5, Sts 71, StW 505) and Swartkrans (SK 46), attributed, respectively, to Australopithecus africanus and Paranthropus robustus..$^{10-14}$ In the absence of well-preserved South African early Pleistocene human crania, we included the Middle Pleistocene composite cranium DH 1/DH 3 from Rising Star attributed to Homo naledi as comparative material for the external morphology. ${ }^{15}$ Additionally, we selected bone thickness values of nine human and non-human Pliocene and Pleistocene hominin taxa presented in the supplementary information of Copes and Kimbel ${ }^{16}$. Our extant comparative sample comprises adult humans (Homo sapiens, $n=10$ ) and common chimpanzees (Pan troglodytes, $n=10$ ) with equal proportions of male and female individuals within each taxon from the collections of the University of Pretoria ${ }^{17}$ (South Africa) and the Royal Museum for Central Africa (Belgium) respectively. Ethical clearance for the use of extant human crania was obtained from the Main Research Ethics Committee of the Faculty of Health Sciences, University of Pretoria in February 2016 (35/2016).

The new fossils, StW 671 and StW 672, were scanned at the microfocus X-ray tomography facility of the Palaeosciences Centre at the University of the Witwatersrand, in Johannesburg (South Africa), at a spatial resolution of $28 \mu \mathrm{m}$ (isotropic voxel size) (Figures 1 and 2). Fossil comparative material from Sterkfontein and Swartkrans as well as extant specimens were scanned at the Palaeosciences Centre, at the South African Nuclear Energy Corporation in Pelindaba (South Africa), or at the Centre for X-ray Tomography of the Ghent University in Ghent (Belgium). Additionally, for external morphology, we included in our study the digital replica of the composite cranium $\mathrm{DH}$ 1/DH 3 from Rising Star available on MorphoSource (www.morphosource.org). ${ }^{15}$

StW 671 and StW 672 were digitally rendered with Avizo v.9.0 software (Visualization Sciences Group Inc.). We extracted one section in StW 671 and one section in StW 672, sampling maximum length/width of the preserved cranial fragments (Figure 2). In terms of measurements, we followed the protocol detailed in Beaudet et al. ${ }^{18}$ In view of collecting consistent data throughout the sample, we tried to avoid oversampling exocranial reliefs and, thus, the section in StW 671 was positioned orthogonally to the temporal line. The three layers of the bone were segmented by combining manual and automatic methods (i.e. watershed transform). Thickness and surface area of the inner table of the diploë and

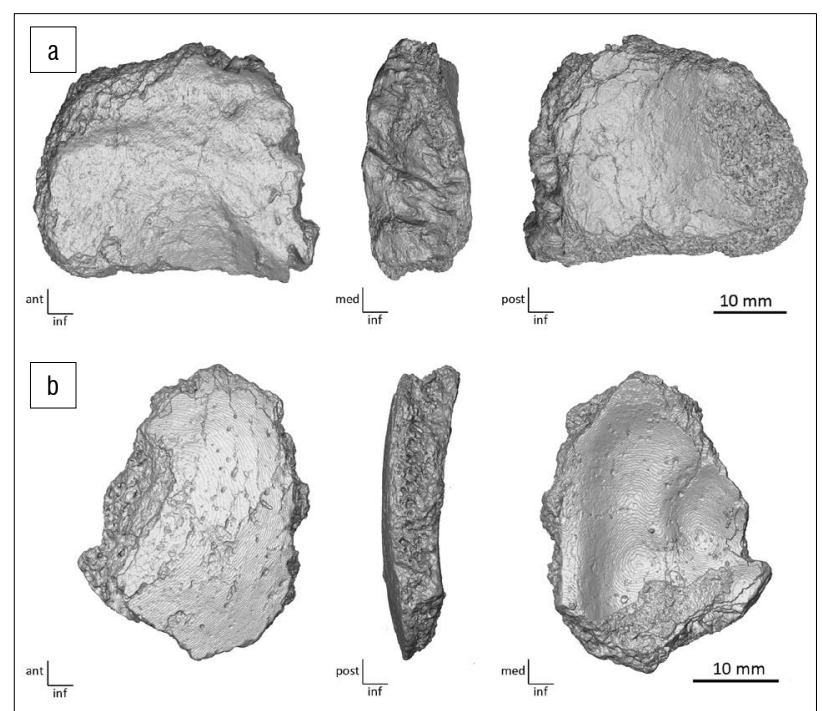

Figure 1: Virtual rendering of (a) StW 671 and (b) StW 672 in ectocranial (left), posterior/medial (middle) and endocranial (right) views. of the outer table were automatically and separately measured at regular intervals along the two sections perpendicular to the outer cranial surface using a custom-written program in MATLAB R2013a ${ }^{18}$ (Mathworks, https://www.mathworks.com/products/matlab.html) (Figure 2). In total, 30 measurements were collected on StW 671 and on StW 672. Based on surface area, we computed bone tissue proportions as the percentage of the bone area represented by outer/inner tables or diploë..$^{18}$ For comparative specimens, with the exception of $\mathrm{DH} 1 / \mathrm{DH} 3$, we selected a number of measurements (see Results) performed along parasagittal sections from Beaudet et al..$^{18}$ and corresponding to the portions of the cranium documented in StW 671 and StW 672.

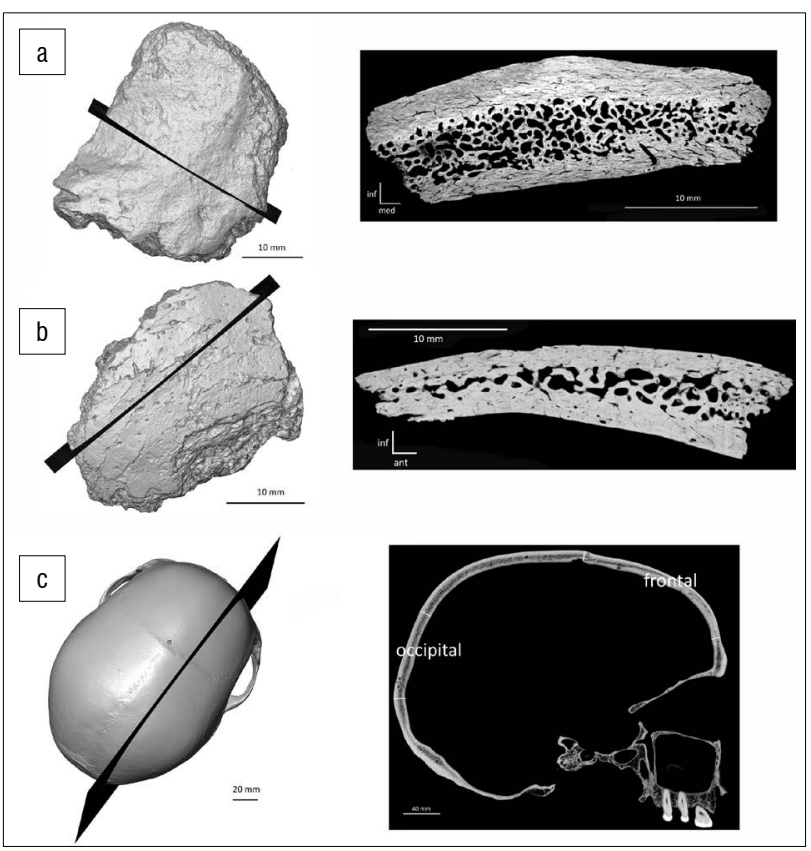

Figure 2: Cranial structural organisation along sections extracted from (a) StW 671 and (b) StW 672. Comparative measurements come from (c) the frontal and occipital parts of the parasagittal section extracted from the comparative sample. White lines in (c) delimitate the frontal and occipital portions of the cranial vault.

\section{Results}

\section{Description of StW 671 and StW 672}

StW 671 is a small rectangular fragment of the frontal bone (maximum length $=38 \mathrm{~mm}$; maximum width $=32.5 \mathrm{~mm}$; maximum intertabular thickness $=13.7 \mathrm{~mm}$ ) (Figure 1a). On the lateral aspect, the temporal line runs along the ectocranial surface. The posterior border of the fragment corresponds to the coronal suture. The surface immediately inferior to the temporal line is relatively flat. The endocranial surface is convex and does not exhibit any diagnostic anatomical features. In terms of surface preservation, StW 671 shows a few minor weathering cracks and heavy manganese dioxide staining.

StW 672 is an ovoid fragment of the occipital bone (maximum length = $34.3 \mathrm{~mm}$; maximum width $=25.1 \mathrm{~mm}$; maximum thickness $=7.4 \mathrm{~mm}$ ) (Figure 1b). The ectocranial surface is relatively smooth. The endocranial surface preserves two shallow grooves and a faint ridge is perceptible along the medial border. The fragment is globally convex. Because the ectocranial and endocranial surfaces lack crests and vascular grooves, respectively, this fragment is proposed to originate from the portion of the occipital bone superior to the nuchal crest and transverse sinus. The specimen's surface shows minor weathering, including cracks, and evidence of diagenetic flaking in the form of non-morphological pits on the ectocranial aspect. 


\section{Comparative description of StW 671 and StW 672}

StW 671 and StW 672 were superimposed on the digital replica of Sts 5 (Australopithecus africanus), SK 46 (Paranthropus robustus), a composite skull of Homo naledi based on $\mathrm{DH} 1$ and $\mathrm{DH} 3$, and extant human and chimpanzee crania (Figure 3).

The overall morphology of StW 671 does not fit well with the external aspect of the frontal bone of Sts 5 , SK 46, DH 1/DH 3 and of the chimpanzee specimen. Indeed, when superimposed onto Sts 5 and DH 1/DH 3, the portion of the frontal bone above the temporal line in StW 671 is more elevated than in the comparative frontal squama. The temporal line progressively joins the sagittal crest in Paranthropus and chimpanzees. ${ }^{19}$ If the temporal line of StW 671 is superimposed onto the temporal line of SK 46 and of the chimpanzee specimen, the coronal suture in the Milner Hall specimen is then positioned in the middle of the frontal bone of the two comparative specimens. The overall morphology of the StW 671 fragment closely fits the shape of the frontal bone in the extant human specimen. However, when superimposed, the temporal line in StW 671 runs inferiorly compared to the human cranium.

The morphology of StW 672 is compatible with the morphology of the occipital bone for all comparative crania considered in this study.

\section{Cranial thickness and composition in StW 671 and StW 672}

Thickness values and tabular proportions in StW 671 and StW 672 as well as in the comparative sample are shown in Tables 1 and 2 and in Figure 4. From Beaudet et al. ${ }^{18}$, we specifically selected measurements 1-20 and 40-50, respectively representing the frontal and occipital bones (Figure 2c). Additionally, we compiled comparative measurements of the frontal bone of non-human and human hominin taxa from Copes and Kimbel ${ }^{16}$ in Table 3.

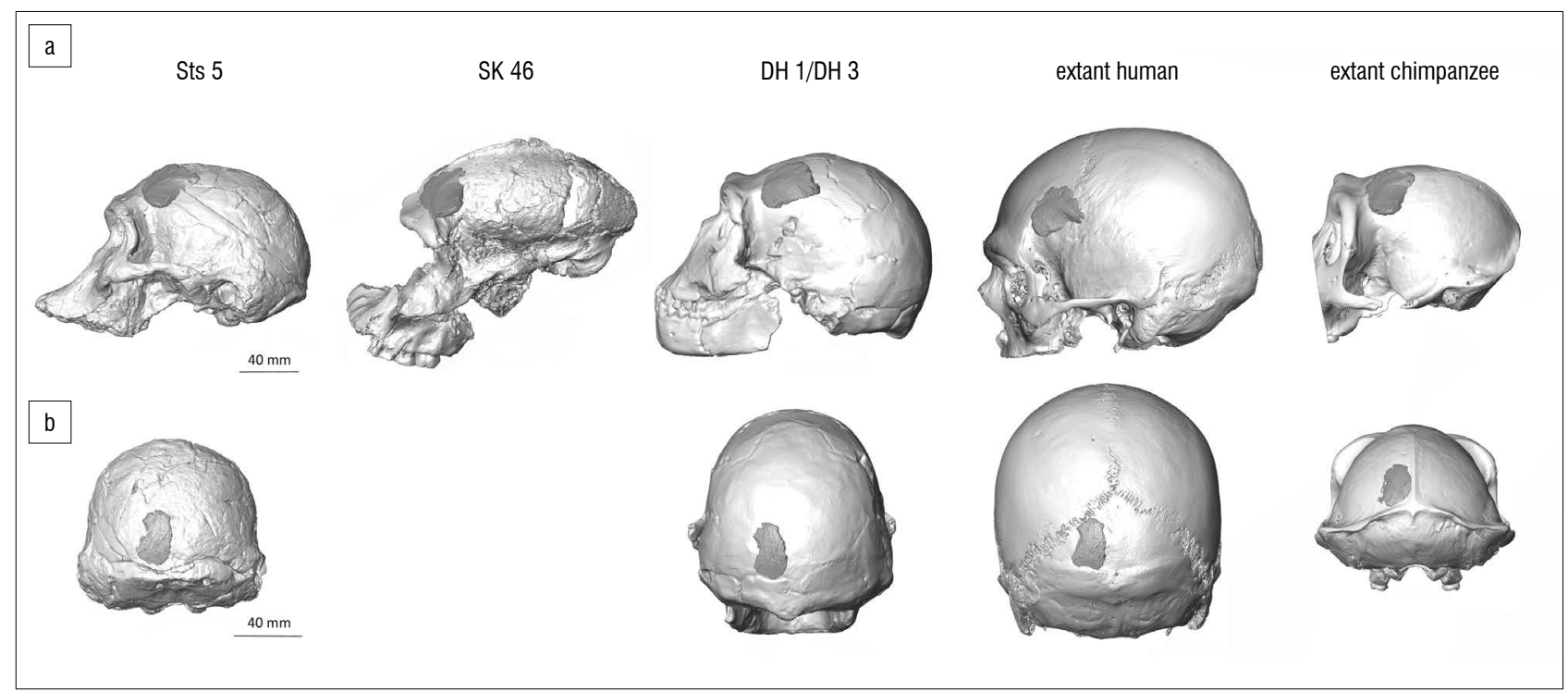

Figure 3: Superimposition of (a) StW 671 (in lateral view) and (b) StW 672 (in posterior view) on the digital replicas of Sts 5 (Australopithecus africanus), SK 46 (Paranthropus robustus), DH 1/DH 3 (Homo naledi) and extant human and chimpanzee crania.

Table 1: Mean frontal bone thickness (FBT, $\mathrm{mm}$ ) and relative proportions of the diploë (\%) of StW 671 compared to the estimates from some Plio-Pleistocene hominin specimens and extant humans and chimpanzees. Standard deviations are shown in brackets.

\begin{tabular}{l|c|c|c|c|c}
\hline \hline & FBT & $\begin{array}{c}\text { Outer } \\
\text { table }\end{array}$ & $\begin{array}{c}\text { Inner } \\
\text { table }\end{array}$ & Diploë & \% Diploë \\
\hline \hline StW 671 & $8.8(0.9)$ & $2.9(0.3)$ & $1.8(0.4)$ & $4.1(0.8)$ & $45.1(5.9)$ \\
\hline StW 505 & $10.2(5.3)$ & - & - & - & - \\
\hline Sts 5 & $6.3(3.1)$ & $0.2(0.3)$ & $1.1(0.6)$ & $5.0(2.5)$ & $77.6(4.1)$ \\
\hline Sts 71 & $7.8(1.1)$ & $0.9(0.2)$ & $0.6(0.3)$ & $6.3(1.2)$ & $78.7(5.3)$ \\
\hline SK 46 & $7.4(4.2)$ & $7.1(4.2)^{\star}$ & $0.3(0.5)$ & $6.4(8.9)$ \\
\hline $\begin{array}{l}\text { Extant humans } \\
(n=10)\end{array}$ & $7.6(0.7)$ & $1.9(0.4)$ & $1.7(0.2)$ & $4.1(0.8)$ & $51.0(5.9)$ \\
\hline $\begin{array}{l}\text { Extant } \\
\text { chimpanzees } \\
(n=10)\end{array}$ & $4.6(1.0)$ & \multicolumn{2}{|c|}{$3.7(0.5)^{\star}$} & $1.0(0.8)$ & $15.6(4.7)$ \\
\hline
\end{tabular}

* The outer and inner tables are indistinct (i.e. cortical) in SK 46 and extant chimpanzees.
Table 2: $\quad$ Mean occipital bone thickness (OBT, $\mathrm{mm}$ ) and relative proportions of the diploë (\%) of StW 672 compared to the estimates from some Plio-Pleistocene hominin specimens and extant humans and chimpanzees. Standard deviations are shown in brackets.

\begin{tabular}{l|c|c|c|c|c}
\hline \hline & OBT & $\begin{array}{c}\text { Outer } \\
\text { table }\end{array}$ & $\begin{array}{c}\text { Inner } \\
\text { table }\end{array}$ & Diploë & \% Diploë \\
\hline \hline StW 672 & $5.6(0.9)$ & $1.4(0.3)$ & $1.8(0.3)$ & $2.4(0.9)$ & $41.3(10.2)$ \\
\hline StW 505 & $9.3(4.9)$ & - & - & - & - \\
\hline Sts 5 & $9.2(4.2)$ & $0.6(0.6)$ & $0.9(0.3)$ & $7.7(3.9)$ & $83.4(35.6)$ \\
\hline Sts 71 & $7.0(2.9)$ & $0.2(0.3)$ & $0.3(0.2)$ & $6.4(2.8)$ & $89.5(43.6)$ \\
\hline SK 46 & $6.7(1.3)$ & \multicolumn{2}{|c|}{$6.1(1.8)^{\star}$} & $0.6(0.7)$ & $11.2(11.8)$ \\
\hline $\begin{array}{l}\text { Extant humans } \\
(n=10)\end{array}$ & $6.9(0.3)$ & $1.9(0.6)$ & $1.3(0.2)$ & $3.8(0.5)$ & $52.5(7.6)$ \\
\hline $\begin{array}{l}\text { Extant } \\
\text { chimpanzees } \\
(n=10)\end{array}$ & $4.6(0.6)$ & \multicolumn{2}{|c|}{$3.9(0.6)^{\star}$} & $0.8(0.2)$ & $14.8(1.9)$ \\
\hline
\end{tabular}

* The outer and inner tables are indistinct (i.e. cortical) in SK 46 and extant chimpanzees. 
Table 3: Mean frontal bone thickness (FBT, $\mathrm{mm}$ ) of human and nonhuman hominin species from Copes and Kimbel ${ }^{16}$. Standard deviations are shown in brackets.

\begin{tabular}{l|c|c|c|c|c}
\hline \hline & $n$ & FBT & $\begin{array}{c}\text { Outer } \\
\text { table }\end{array}$ & $\begin{array}{c}\text { Inner } \\
\text { table }\end{array}$ & Diploë \\
\hline \hline Australopithecus afarensis & 2 & $7.5(1.8)$ & $1.4(0.4)$ & $1.3(0.3)$ & $6.0(0.3)$ \\
\hline Paranthropus boisei & 7 & $6.0(1.2)$ & - & - & - \\
\hline Early Homo & 10 & $6.0(1.5)$ & - & - & - \\
\hline European Homo erectus & 2 & $8.5(0.7)$ & - & - & - \\
\hline African Homo erectus & 8 & $9.2(1.3)$ & - & - & - \\
\hline Asian Homo erectus & 29 & $9.2(2.1)$ & - & - & - \\
\hline Homo heidelbergensis & 14 & $8.5(1.8)$ & - & - & - \\
\hline Homo neanderthalensis & 13 & $6.6(1.6)$ & - & - & - \\
\hline Pleistocene Homo sapiens & 17 & $7.5(2.1)$ & - & - & - \\
\hline
\end{tabular}

The mean frontal bone thickness in StW 671 is thick compared to Sts 5 , Sts 71 , SK 46 and the extant human and chimpanzee specimens with the exception of StW 505 (Table 1, Figure 4a). When compared to the frontal bone thickness values reported by Copes and Kimbel ${ }^{16}$, the closest matches are Homo erectus and Homo heidelbergensis (Table 3). StW 671 shares the following pattern of bone tissue proportions with Sts 71, extant humans and Australopithecus afarensis: the diploë is the thickest bony layer while the inner table is the thinnest (Tables 1 and 3 ). As previously noted ${ }^{20,21}$, Sts 5 lacks a significant portion of its outer table. In terms of tissue proportions (Table 1, Figure 4a), the diploic bone contributes $45.1 \%$ to the cumulative cranial thickness in StW 671, while in Sts 5 , Sts 71 and extant humans the diploë represents more than $50 \%$ of the total thickness and less than $20 \%$ in SK 46 and extant chimpanzees (Figure 4b).

The mean occipital bone thickness in StW 672 is thin compared to the comparative fossil specimens and extant humans (Table 2, Figure 4c). The pattern of bone tissue proportions in the comparative sample is variable but StW 672 and Sts 5 and Sts 71 have a similar pattern in that the diploë is the thickest bony layer while the outer table is the thinnest (Table 2). The proportion of diploë in StW 672 represents $41.3 \%$ of the cumulative cranial thickness and more than $50 \%$ in StW 578, Sts 5, Sts 71 and extant humans but less than $20 \%$ in SK 46 and extant chimpanzees (Figure 4d).

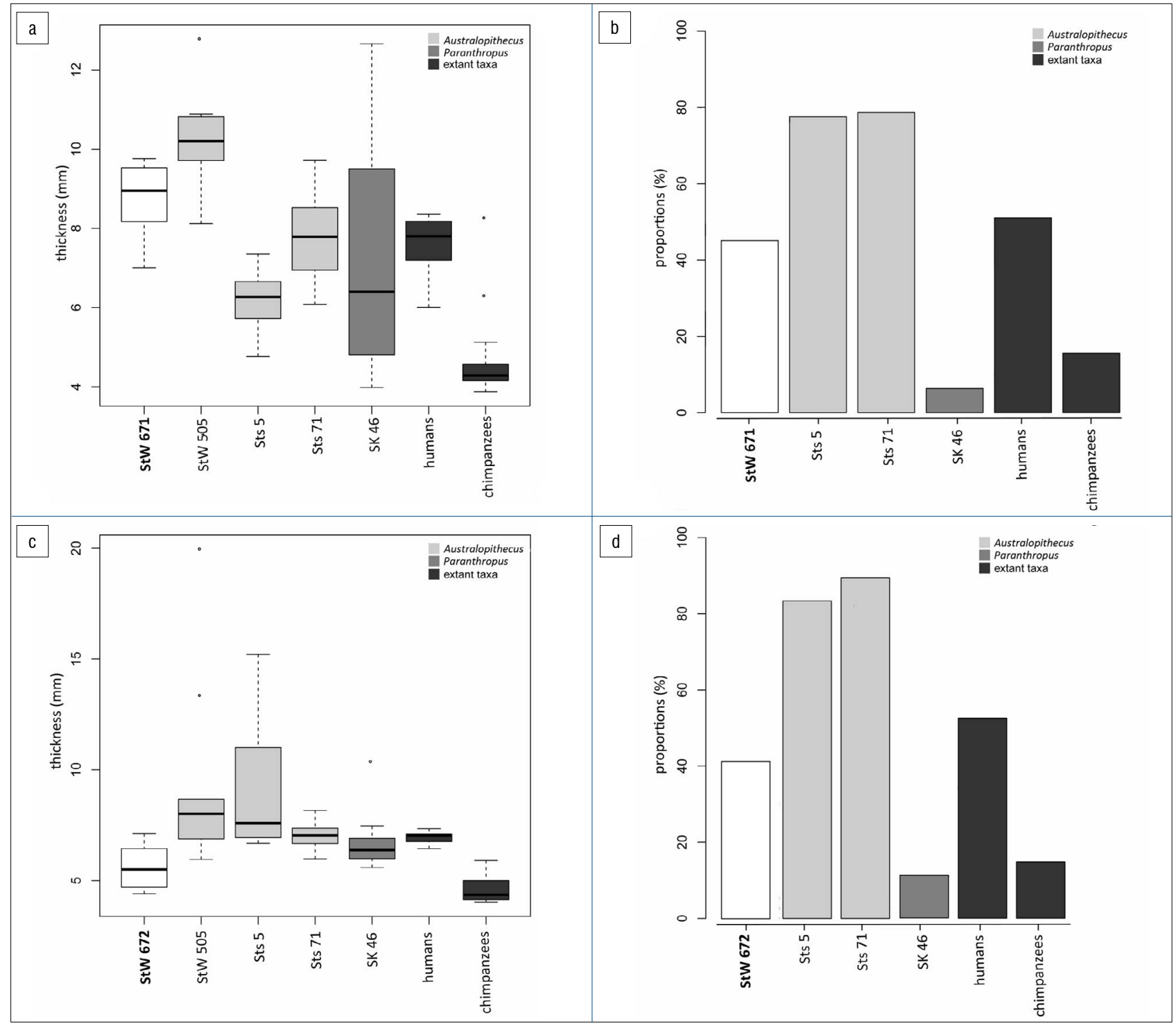

Figure 4: ( $a$ and $c)$ Box plots of cranial thickness and ( $b$ and $d$ ) histograms of proportion of diploë in (a, b) StW 671 and (c, d) StW 672 compared to some Plio-Pleistocene hominin specimens/taxa and extant human crania. 


\section{Discussion}

While the overall morphology and cranial thickness of StW 671 approximates the human condition, the proportion of diploë in both StW 671 and StW 672 is lower than that found in human and nonhuman hominin taxa investigated in this study with the exception of Paranthropus. Interestingly, despite StW 671 showing a derived humanlike cranial morphology and thickness, the proportions of diploë in StW 671 and StW 672, even if comparatively closer to extant humans, do not clearly match the fossil hominins included in our comparative study. In this context, our analysis may suggest a combination of derived humanlike traits and unique features. This mosaicism is compatible with the description and metric analyses of the manual proximal phalanx StW 668 and the upper right first molar StW 669 from Milner Hall and contributes to a certain degree of taxonomic ambiguity. ${ }^{1}$ As the diploic bone acts as a protective barrier for the brain, contributes to mechanical properties of the cranium and plays a role in the cranial vascular system, our results may potentially suggest an intriguing distinct palaeobiology in the Milner Hall hominins. ${ }^{16,22-25}$

Further analyses documenting variation in the frontal anatomy (e.g. postorbital constriction) and cranial thickness in early hominins, and more particularly in South African and East African early Homo, would be critical for the interpretations of the Milner Hall hominin palaeobiology and taxonomic attribution. Moreover, comparisons with the East African early Homo material preserving frontal and occipital bones (e.g. KNM-ER 1470, KNM-ER 1813) would be particularly relevant for supporting the presence of human-like traits in the Milner Hall hominins' anatomy and/or assessing the potential uniqueness of the $\mathrm{MH}$ fossil record. Additionally, because the position of our sections might vary across specimens, our bidimensional quantitative investigation of the cranial bone thickness and composition should be combined in future with 3D approaches ${ }^{16}$ and/or supported by new findings of more complete cranial fragments from the Milner Hall deposits. Nevertheless, besides reporting additional specimens from STK-MH1, this study highlights the relevance of the analysis of cranial thickness and composition in taxonomical studies and the potential of the Milner Hall for contributing to our knowledge of the hominin palaeobiodiversity at Sterkfontein.

\section{Acknowledgements}

We are indebted to E. Gillisen and W. Wendelen (Tervuren), G. Krüger (Pretoria), L. Kgasi, H. Fourie, S. Potze and M. Tawane (Pretoria) and B. Zipfel (Johannesburg) for having granted access to fossil and comparative material under their care. We also thank L. Bam, F. de Beer and J. Hoffman (Pelindaba), M. Dierick (Ghent) and K. Jakata (Johannesburg) for microtomographic acquisitions. We are grateful to the Ditsong National Museum of Natural History and the University of the Witwatersrand for loaning hominin crania in their collection. For technical and/or scientific discussion/collaboration we are grateful to: M. Carmen Arriaza (Johannesburg), R. Clarke (Johannesburg), J. Dumoncel (Toulouse), K. Carlson (Los Angeles) and A. Oettlé (Pretoria). We thank the DST-NRF for sponsoring the Micro-XCT facility at Necsa, and the DST-NRF and the University of the Witwatersrand for funding the microfocus X-ray CT facility in the ESI (www.wits.ac.za/microct). The support of the AESOP + Programme, the Claude Leon Foundation, the DST-NRF Centre of Excellence in Palaeosciences, the French Institute of South Africa and the Palaeontological Scientific Trust (PAST) towards this research is hereby acknowledged. Opinions expressed and conclusions arrived at, are those of the authors and are not necessarily to be attributed to the Centre of Excellence in Palaeosciences.

\section{Authors' contributions}

Conceptualisation: A.B., T.R.P., J.L.H., D.S.; methodology: A.B., E.N.L.; data collection: A.B., D.S., T.R.P., J.L.H.; sample and data analysis: A.B., E.N.L., T.R.P., J.L.H.; validation: T.R.P., J.L.H., E.N.L.; writing: A.B., D.S., T.R.P., J.L.H., E.N.L.; project leadership: D.S.; project management: A.B.; funding acquisition: A.B., D.S.

\section{References}

1. Stratford D, Heaton JL, Pickering TR, Caruana MV, Shadrach K. First hominin fossils from Milner Hall, Sterkfontein, South Africa. J Hum Evol. 2016;91:167-173. https://doi.org/10.1016/j.jhevol.2015.12.005

2. Stratford DJ, Grab S, Pickering TR. The stratigraphy and formation history of fossil-and artefact-bearing sediments in the Milner Hall, Sterkfontein Cave, South Africa: New interpretations and implications for palaeoanthropology and archaeology. J Afr Earth Sci. 2014;96:155-167. https://doi.org/10.1016/j. jafrearsci.2014.04.002

3. Granger DE, Gibbon RJ, Kuman K, Clarke RJ, Bruxelles L, Caffee MW. New cosmogenic burial ages for Sterkfontein Member 2 Australopithecus and Member 5 Oldowan. Nature. 2015;522:85-88. https://doi.org/10.1038/ nature 14268

4. Clarke RJ. Australopithecus from Sterkfontein Caves, South Africa. In: Reed K, Fleagle JG, Leakey RE, editors. The paleobiology of Australopithecus. Dordrecht: Springer; 2013. p. 105-123. https://doi.org/10.1007/978-94007-5919-0

5. Grine FE. The alpha taxonomy of Australopithecus africanus. In: Reed KE, Fleagle JG, Leakey RE, editors. The paleobiology of Australopithecus. Dordrecht: Springer; 2013. p. 74-104. https://doi.org/10.1007/978-94-0075919-0

6. Grine FE, Smith HF, Heesey CP, Smith EJ. Phenetic affinities of PlioPleistocene Homo fossils from South Africa: Molar cusp proportions. In: Grine FE, Fleagle JG, Leakey RE, editors. The first humans: Origin and early evolution of the genus Homo. Dordrecht: Springer; 2009. p. 49-62. https:// doi.org/10.1007/978-1-4020-9980-9

7. Curnoe D. A review of early Homo in southern Africa focusing on cranial, mandibular and dental remains, with the description of a new species (Homo gautengensis sp nov.). Homo. 2010;61:151-177. https://doi.org/10.1016/j. jchb.2010.04.002

8. Clarke RJ. Homo habilis: The inside story. In: Sahnouni M, Semaw S, Garaizar JR, editors. Proceedings of the II Meeting of African Prehistory. Burgos: Consorcio CENIEH; 2017. p. 25-51.

9. Clarke RJ. The cranium of the Swartkrans hominid, SK 847 and its relevance to human origins [PhD thesis]. Johannesburg: University of the Witwatersrand; 1977.

10. Broom R. Discovery of a new skull of the South African ape-man, Plesianthropus. Nature. 1947;159:672. https://doi.org/10.1038/159672a0

11. Broom R, Robinson JT. Further evidence of the structure of the Sterkfontein ape-man Plesianthropus. Transv Mus Mem. 1950;4:7-83.

12. Broom R, Robinson JT. Swartkrans ape-men. Paranthropus crassidens. Pretoria: Transvaal Museum; 1952.

13. Broom R, Robinson JT, Schepers GWH. Sterkfontein ape-man, Plesianthropus. Transv Mus Mem. 1950;4:1-117.

14. Lockwood CA, Tobias PV. A large male hominid cranium from Sterkfontein, South Africa, and the status of Australopithecus africanus. J Hum Evol. 1999;36:637-685. https://doi.org/10.1006/jhev.1999.0299

15. Berger LR, Hawks J, De Ruiter DJ, Churchill SE, Schmid P, Delezene LK, et al. Homo naledi, a new species of the genus Homo from the Dinaledi Chamber South Africa. eLife. 2015; 4, e09560, 35 pages. https://doi.org/10.7554/ elife.09560

16. Copes LE, Kimbel WH. Cranial vault thickness in primates: Homo erectus does not have uniquely thick vault bones. J Hum Evol. 2016;90:120-134. https://doi.org/10.1016/j.jhevol.2015.08.008

17. L'Abbé EN, Loots M, Meiring JH. The Pretoria Bone Collection: A modern South African skeletal sample. Homo. 2005;56:197-205. https://doi. org/10.1016/j.jchb.2004.10.004

18. Beaudet A, Carlson KJ, Clarke RJ, De Beer F, Dhaene J, Heaton JL, et al. Cranial vault thickness variation and inner structural organization in the StW 578 hominin cranium from Jacovec Cavern, South Africa. J Human Evol. 2018;121:201-220. https://doi.org/10.1016/j.jhevol.2018.04.004

19. Aiello L, Dean C. An Introduction to human evolutionary anatomy. New York: Academic Press; 1990. https://doi.org/10.1016/C2009-0-02515-X

20. Wolpoff MH. Sagittal cresting in the South African australopithecines. Am J Phys Anthropol. 1974;40:367-408. https://doi.org/10.1002/ajpa.1330400312 
21. Thackeray JF. Cranial bone of 'Mrs Ples' (Sts 5): Fragments adhering to matrix. S Afr J Sci. 1997;93:169-170.

22. McElhaney JH, Fogle JL, Melvin JW, Haynes RR, Roberts VL, Alem NM. Mechanical properties of cranial bone. J Biomech. 1970;5:495-496. https:// doi.org/10.1016/0021-9290(70)90059-X

23. Hershkovitz I, Greenwald C, Rotschild B, Latimer B, Dutour O, Jellema LM, et al. The elusive diploic veins: Anthropological and anatomical perspective. Am J Phys Anthropol. 1999;108:345-358. https://doi.org/10.1002/(SICI)10968644(199903)108:3<345::AID-AJPA9>3.0.C0;2-S
24. Anzelmo M, Ventrice F, Barbeito-Andres J, Pucciarelli HM, Sardi ML. Ontogenetic changes in cranial vault thickness in a modern sample of Homo sapiens. Am J Hum Biol. 2015;27:475-485. https://doi.org/10.1002/ ajhb.22673

25. Rangel de Lázaro G, De la Cuétara JM, Pišová H, Lorenzo C, Bruner E. Diploic vessels and computed tomography: Segmentation and comparison in modern humans and fossil hominids. Am J Phys Anthropol. 2016;159:313324. https://doi.org/10.1002/ajpa.22878 